Roux Jean-Claude, La Bolivie orientale. Confins inexplorés, battues aux Indiens et économie de pillage (1825-1992), L'Harmattan, Paris, 2000, 316 p., bibl., ill., cartes, tabl., index

\title{
Mickael Brohan
}

\section{(2) OpenEdition}

\section{Journals}

Édition électronique

URL : https://journals.openedition.org/jsa/2923

DOI : 10.4000/jsa.2923

ISSN : 1957-7842

Éditeur

Société des américanistes

Édition imprimée

Date de publication : 5 janvier 2005

Pagination : 242-245

ISSN : 0037-9174

Référence électronique

Mickael Brohan, « Roux Jean-Claude, La Bolivie orientale. Confins inexplorés, battues aux Indiens et économie de pillage (1825-1992), L'Harmattan, Paris, 2000, 316 p., bibl., ill., cartes, tabl., index », Journal de la Société des américanistes [En ligne], 91-1 | 2005, mis en ligne le 04 octobre 2006, consulté le 04 septembre 2022. URL : http://journals.openedition.org/jsa/2923 ; DOl : https://doi.org/10.4000/jsa. 2923

Ce document a été généré automatiquement le 4 septembre 2022.

Tous droits réservés 


\title{
Roux Jean-Claude, La Bolivie orientale. Confins inexplorés, battues aux Indiens et économie de pillage (1825-1992), L'Harmattan, Paris, 2000, 316 p., bibl., ill., cartes, tabl., index
}

\author{
Mickael Brohan
}

1 L'Orient bolivien, qui s'étend des forêts du bas piémont et de haute Amazonie jusqu'aux savanes du Chaco en passant par les plaines du Beni, la région de Santa Cruz et le massif de la Chiquitania, représente plus de la moitié du territoire national. En dépit de son immense superficie (presque $700000 \mathrm{~km}^{2}$ ), cet espace tardivement colonisé est toutefois demeuré longtemps méconnu et marginal(isé) au sein de ce pays résolument tourné vers les hautes terres andines. Aussi, bien qu'elle soit depuis peu l'objet d'une attention croissante, visible dans la plupart des domaines de la recherche en sciences humaines (histoire, géographie, anthropologie, sociologie, linguistique), cette vaste zone est encore largement peu étudiée. Quelle que soit leur discipline de prédilection, les spécialistes de la Bolivie en général, de l'Amazonie et du Chaco en particulier, accueilleront avec intérêt la publication de cet ouvrage de géo-histoire et de géopolitique sur cette région.

Qu'ils ne s'en réjouissent néanmoins pas trop avant lecture car, avouons-le, ce livre de Jean-Claude Roux qui propose une étude de la construction territoriale, politique et économique de l'Orient bolivien depuis l'Indépendance jusqu'à nos jours est très franchement décevant. L'objet est certes prometteur, mais son traitement s'avère, hélas, insuffisant : la plupart des conclusions de l'auteur sur le XIX ${ }^{e}$ siècle sont déjà connues, comme le sont celles établissant, dans la troisième partie, un lien de cause à effet entre l'incapacité bolivienne à définir et, surtout, à appliquer une véritable politique de colonisation orientale et le démembrement du territoire national, opéré essentiellement dans les confins orientaux du pays (pertes successives du futur Acre, du Mato Grosso, du 
Purus et du Chaco boréal). Quant au $\mathrm{xx}^{\mathrm{e}}$ siècle, il n'est évoqué que dans un trop bref épilogue d'à peine vingt-cinq pages alors que la question du développement multiforme et inégal d'un surprenant dynamisme dans cette région aurait mérité un examen moins superficiel. Par ailleurs, s'il procède d'un réel effort de recherche des sources d'époque (on conseillera d'aller voir en détail la volumineuse bibliographie de presque quinze pages ${ }^{1}$ ) et d'une évidente volonté de pluridisciplinarité, cet ouvrage témoigne pourtant d'une non-maitrise par l'auteur de cette volumineuse littérature et de l'échec de ses incursions dans les disciplines indispensables pour cerner l'histoire de l'Orient bolivien nous pensons en particulier à l'ethnologie et à la sociologie. Ces faiblesses se traduisent notamment par une méconnaissance totale des sociétés indigènes peuplant l'espace étudié, par un manque de distance flagrant avec les sources utilisées et par des erreurs en cascade. Épineux problème pour un géographe, cette contribution présente aussi une cartographie souvent très approximative. En somme, on retrouve ici les mêmes déficiences que celles qui sont signalées par Philippe Erikson à propos d'un autre livre de ce même auteur sur L'Amazonie péruvienne ${ }^{2}$ dont celui sur La Bolivie orientale peut être perçu comme le pendant.

3 Penchons-nous en premier lieu sur le traitement réservé aux groupes indigènes qui occupent logiquement une place de choix dans le corps du texte. Outre l'emploi à leur égard d'un vocabulaire d'un autre âge tout à fait surprenant dans une publication aux ambitions scientifiques («barbares», p.17; «sauvages», p. 225 !), les références ethnologiques relatives aux questions évoquées sont quasi systématiquement ignorées par l'auteur, ce qui affaiblit considérablement son exposé : la connaissance de deux ouvrages sur les divers mouvements messianiques des Mojeños ${ }^{3}$ lui aurait par exemple permis d'éviter une analyse caricaturale et l'utilisation d'une expression malheureuse ("d'obscurs messies", p. 18). Lorsqu'il se risque à citer certains travaux, ceux-ci sont souvent anciens : par exemple, sur la complexe ethnogénèse chiriguano, seuls les écrits d'Alfred Métraux et d'Erland Nordenskiöld sont évoqués (p. 36), alors que, pour s'en tenir à la littérature francophone, Isabelle Combès et Thierry Saignes ont livré, il y a une quinzaine d'années, une célèbre étude sur cette question ${ }^{4}$. Aussi, dans les très rares cas où les ouvrages mentionnés font réellement autorité, on peut sérieusement se demander s'ils ont été lus car, sinon, comment expliquer notamment le fait que Jean-Claude Roux insiste à plusieurs reprises sur l'absence de relations humaines et culturelles entre les aires andine et amazonienne de l'actuelle Bolivie aux temps pré-coloniaux (pp. 33, 35, 70) tout en citant (mal) dans sa traduction espagnole le fameux ouvrage collectif de RenardCasevitz et al. ${ }^{5}$, lequel démontre précisément le contraire? Du reste, alors qu'un simple coup d'œil sur l'un des nombreux guides ethniques régionaux aurait suffi à éviter ces erreurs, les ethnonymes sont ici quasi systématiquement déformés (Ipuranas au lieu de Ipurina, p. 225 ; Aromas au lieu de Araona, p. 218 ; Toronomas au lieu de Toromona, idem ; Nachtenes au lieu de Noctene, p. 105, etc.). Par ailleurs, on cherchera en vain la logique poussant Jean-Claude Roux à employer ces ethnonymes quelquefois à la mode espagnole (au pluriel) et quelquefois à la mode française (au singulier) et l'on s'étonnera de la francisation complète et arbitraire de l'un d'entre eux (Cavinais au lieu de Cavineño, p. 232). Enfin, les cartes ethniques sont non seulement incomplètes, mais aussi d'une grande imprécision géographique quant à la localisation des sociétés indigènes que, par ailleurs, on ne parvient pas toujours à identifier. Celle du "Far East de Fitzcarrald » (p. 246) ne fait état, à la fin du xix ${ }^{\mathrm{e}}$ siècle, dans la zone allant du moyen Madre de Dios jusqu'au haut Ucayali, que de la présence des Cashibos et des Mashcos (qui sont situés très approximativement) ainsi que des énigmatiques Shahuintos (sic) et Uncunini (sic)! 
Quant à la carte des groupes du Chaco (p. 105), l'auteur s'abstient tout simplement de préciser l'époque dont elle est censée rendre compte. Il n'y a pas que l'aspect ethnique de la cartographie qui pose problème. L'échelle y est trop souvent oubliée ; sans prétendre être spécialiste de la question, le tracé du réseau hydrographique présenté nous semble des plus étonnants ou, encore, à de nombreuses occasions, le nom des fleuves est incorrect (Madi au lieu de Madidi p. 218; Cashpaigli au lieu de Cashpajali, Seri ali au lieu de Serjali, Bulileja au lieu de Sotileja, p. 246...).

Autre problème récurrent dans ce livre : le manque, voire l'absence, de distance critique vis-à-vis des sources utilisées. Sans faire montre d'une vision d'ensemble, l'auteur extrait d'une abondante littérature des informations qu'il empile et reprend comme telles, alors qu'elles se contredisent souvent. Mentionnons un seul exemple parmi bien d'autres qui a retenu notre attention, car il traite de la société indigène sur laquelle nous travaillons. Paraphrasant Ballivian, Roux reprend son idée selon laquelle les Blancs ont appris l'existence des indiens Araona pendant le boum du caoutchouc (p. 215) et qu'importe si, une centaine de pages auparavant, il indiquait qu'un missionnaire les avait visités plus tôt (p. 113) et, surtout, si plusieurs des références citées au fil des pages (Armentia, Maurtua, etc.) attestent d'un contact déjà pluriséculaire à cette époque.

Quant aux innombrables erreurs et coquilles (Foucauld au lieu de Foucault, Bonpland au lieu de Bompland, Ribière au lieu de Rivière, Macchetti au lieu de Machetti, Nordenskjöld ou Nordenskjôld au lieu de Nordenskiöld, Iziamas au lieu de Ixiamas, mallocas au lieu de malocas... ; même le nom de l'institution de l'auteur qui a pourtant apporté son concours à cette publication est mal orthographié en quatrième de couverture!), elles rendent la lecture difficile, voire parfois pénible. Outre ces sérieux défauts, Jean-Claude Roux n'indique jamais les pages des sources et travaux qu'il cite ou paraphrase.

6 À qui parviendrait à faire abstraction de ces déficiences, nous recommandons néanmoins la lecture des quatrième et cinquième parties de l'ouvrage, consacrées respectivement aux économies extractives de la quina (1830-1880) et du caoutchouc (1880-1920) et aux conséquences de la longue absence de l'État bolivien dans cette zone éloignée du pouvoir politique central qu'est l'Oriente. Non pas qu'ils constituent un apport décisif sur ces thèmes encore insuffisamment étudiés - les spécialistes n'y apprendront probablement pas grand-chose-, mais ils ont le mérite de compiler des données dispersées. Dans le chapitre sur les boums de la quina et de la goma, qui ont profondément et durablement transformé le paysage géographique, économique et socioculturel de l'orient (et tout particulièrement de l'actuel département de Pando), Jean-Claude Roux brosse un tableau réaliste et assez fidèle aux sources de cette période trouble qui vit cohabiter, souvent pour le pire, des aventuriers étrangers, des affairistes, des voyageurs naïfs, des paysans trompés et des indigènes amazoniens "esclavagisés » et en voie de "détribalisation " accélérée. Quant au chapitre sur les persistantes insuffisances étatiques dans la région, il présente l'avantage de rappeler en détail des faits souvent oblitérés ou, pour le moins, minimisés dans l'historiographie de l'Orient bolivien. En effet, malgré la faiblesse de l'analyse du système néo-esclavagiste de l'enganche - dont deux de ses caractéristiques essentielles ont été oubliées: la transmission héréditaire de la dette et le passage fréquent et obligé de la main d'œuvre d'un patron à l'autre -, il expose les conditions effroyables de travail auxquelles étaient soumis, lors de la fièvre du caoutchouc, les travailleurs des barracas dont les Indiens, victimes de battues pour compenser la rapide pénurie de la main d'œuvre paysanne et métisse. Par ailleurs, il n'aurait sans doute pas 
été superflu de mentionner que ce système d'exploitation a eu cours (sous différents noms) dans l'ensemble de l'Amazonie et qu'il y perdure aujourd'hui encore en certains endroits ${ }^{6}$.

\section{NOTES}

1.. Jean-Claude Roux est par ailleurs l'auteur d'un ouvrage bibliographique sur les sources relatives à l'Orient bolivien des $\mathrm{XIX}^{\mathrm{e}}$ et $\mathrm{XX}^{\mathrm{e}}$ siècles, intitulé Les Orients boliviens : de l'Amazonie au Chaco, Ed. CRET, université de Bordeaux, Bordeaux, 2002. La littérature proprement ethnologique et linguistique y étant cependant absente, on pourra consulter avec profit les ouvrages de Hans Van der Berg, Bibliografía de las etnias del Oriente boliviano, Universidad católica boliviana, Cochabamba, 1998, et Bibliografía de las lenguas indígenas del Oriente boliviano, Universidad católica boliviana, Cochabamba, 1998.

2.. Jean-Claude Roux, L'Amazonie péruvienne, un El Dorado dévoré par la forêt, 1821-1910, L'Harmattan, Paris, 1994. Voir le compte rendu rédigé par Philippe Erikson dans le Journal de la Société des Américanistes, 1997, 83, pp. 359-360.

3.. Voir, notamment, Jürgen Riester, En busca de la Loma Santa, Los Amigos del Libro, La Paz-Cochabamba, 1976, et Zulema Lehm, Milenarismo y movimientos sociales en la Amazonia boliviana. La busqueda de la Loma Santa y la marcha indígena por el territorio y la dignidad, Ciddebeni-Apcob-Oxfam América, Santa Cruz, 1999. Voir également le compte rendu rédigé par mes soins dans le Bulletin de l'Institut français d'études andines, 2004, 33 (2), pp. 398-402.

4.. Isabelle Combès et Thierry Saignes, Alter ego. Naissance de l'identité chiriguano, Éd. EHESS, Paris, 1991. Voir également le compte rendu rédigé par Isabelle Daillant dans L'Homme, 1992, 122-124, pp. 436-440.

5.. France-Marie Renard-Casevitz, Thierry Saignes et Anne-Christine Taylor, Al este de los Andes, Institut français d'études andines / Abya Yala, Lima / Quito, 1988 [1986].

6.. Le lecteur intéressé trouvera un bon examen de ce système, réalisé à partir d'une étude de cas récente sur son importance dans l'Amazonie brésilienne contemporaine, dans l'ouvrage du regretté Christian Geffray, Chronique de la servitude en Amazonie brésilienne. Essai sur l'exploitation paternaliste, Karthala, Paris, 1995.

\section{AUTEURS}

MICKAEL BROHAN

Université Paris X, Nanterre 